\title{
A STUDY ON THE VIRTUAL DIGGING SIMULATION OF A HYDRAULIC EXCAVATOR
}

\author{
$\underline{\text { Young Bum Kim }}^{1}$, Hyuk Kang ${ }^{2}$, Jun Hyeong $\mathrm{Ha}^{2}$, \\ Moo Seung Kim ${ }^{1}$, Pan Young Kim ${ }^{1}$, Ssang Jae Baek ${ }^{3}$, and Jinsoo Park ${ }^{4}$ \\ ${ }^{1}$ Construction Equipment Research Department, Hyundai Heavy Industries, Ulsan, Korea \\ ${ }^{2}$ Robotics Laboratory, Seoul National University, Seoul, Korea \\ ${ }^{3}$ Construction Equipment Development Department, Hyundai Heavy Industries, Ulsan, Korea \\ ${ }^{4}$ Product Development Research Institute, Hyundai Heavy Industries, Ulsan, Korea \\ *Corresponding author(ybkim07@hhi.co.kr)
}

\begin{abstract}
The interest in unmanned excavator is growing more and more for the sake of work efficiency and safety of the operator. It is general understanding that an optimal working path planning and inverse dynamic control should be resolved first for the realization of the unmanned excavator. In this paper, the methods to determine the optimal working path based on minimum torque or time and to simulate digging works tracking on the designed working path are proposed. In case of the minimum torque, the optimal working path is determined to minimize the joint torques of attachments. On the other hand, in case of minimum time, the optimal working path is decided to minimize time duration for one-cycle working considering the hydraulic constraints such as oil flow rate and relief pressure etc. In order to verify the inverse dynamic code used in the optimization and the optimized path, field measurements were carried out for the various parameters such as swing angle, cylinder lengths, and pressures of a swing motor and hydraulic cylinders during digging works. The modified FEE(Fundamental Earthmoving Equation) is adopted for the modeling of soil-tool interaction in digging process and the inverse dynamics with external force such as constant and reducing lifting weights is used in lifting and dumping process. Finally, all the simulated data were compared with the measured data in order to examine the effectiveness of the proposed methods in view of the development of the unmanned excavator.
\end{abstract}

Keywords: Unmanned Excavator, Optimal Path Planning, Inverse Dynamics, Soil-Tool Interaction, Virtual Digging Simulation

\section{INTRODUCTION}

Each year there are numerous fatal accidents associated with excavators but it is certain that accidents in dangerous environments such as soft ground, collapsing building, and steep slope are avoidable for a human operator. A robust unmanned excavator could be a solution for working in dangerous environments and many related researches are also begun. [1] [2]

The first thing to do for the development of an unmanned excavator is an optimal path planning. The optimal path planning is to calculate the trajectory of minimizing joint torques or time duration when initial and final positions are given. The inverse dynamics analysis of excavator motions including digging, lifting, dumping, and returning is needed to calculate joint torques and calculation of bucket tip force considering soil-tool interaction is also required to apply as an external force in inverse dynamics equation. The calculated optimal path can be used for not only objective value to control an unmanned excavator but also input signal to simulate working motions in a virtual environment. By simulating the motions, the evaluation of durability and performance for various design changes is possible on the virtual environment before manufacturing prototype. We can save time and expenses to develop new model of excavator by the virtual simulation. 
In this study we present analysis techniques of inverse dynamics for a typical digging work considering soil-tool interaction and this is verified by measurement data. The optimal path planning is carried out using presented inverse dynamics and virtual digging simulation environments are built to simulate calculated trajectories.

\section{SOIL-TOOL INTERACTION}

To simulate excavator more realistic, we need the survey of soil-tool interaction which is specified three categories; choice of the soil-tool interaction model, soil parameter estimation and digging force calculation. The measurement data during excavation is used for parameter estimation which is optimized to minimize the difference between measurement and calculated digging forces. All process of the soil-tool interaction was summarized as shown in Fig. 1.

\begin{tabular}{|c|c|}
$\begin{array}{c}\text { Measurement } \\
\text { Data }\end{array}$ & $\begin{array}{c}\text { Soil Parameter } \\
\text { Estimation }\end{array}$ \\
\hline $\begin{array}{c}\text { Digging } \\
\text { Force }\end{array}$ & $\begin{array}{c}\text { Soil-Tool } \\
\text { Interaction Model }\end{array}$ \\
$\begin{array}{c}\text { Digging } \\
\text { Motion Data }\end{array}$ \\
\hline
\end{tabular}

Fig.1 Flow chart of soil-tool interaction identification

\subsection{MODIFIED FEE MODEL}

There are many soil-tool interaction models but their origin is almost $\mathrm{FEE}$ (Fundamental Earth Moving Equation) model. [3] FEE model represents soil-tool interaction as five soil parameters $(\delta, \Phi, \beta, \gamma, c)$ which have geometry or physical meaning. In the recent year, revised version of the FEE model has been developed. [4]

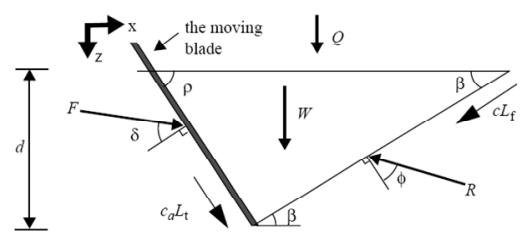

a) The classical soil-tool interaction model(FEE)

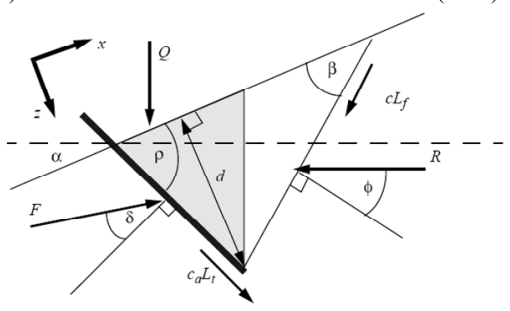

b) The modified FEE model

Fig.2 FEE model classification [4]
As can be seen from Fig.2, we know that modified version can consider slope of the terrain and assume the realistic surcharge Q.

\subsection{ESTIMATION OF SOIL PARAMETERS}

The estimation of soil parameters is important task in soiltool interaction scheme because the digging force is very sensitive to soil parameters. Thus estimation of soil parameters is dominant to simulation accuracy. The estimation of soil parameters was thought as optimization and so we reformulate optimization objective function as below form.

$$
\text { soil parameters }=\arg \min _{\delta, \phi, \gamma, \beta, c} \sum_{i=1}^{n}\left|\Delta F_{i}\right|
$$

The objective variables are soil parameters and objective function is sum of absolute value of force differences. If there are no force differences, the soil-tool interaction model perfectly reflects real soil property. If we can find most optimal soil parameters, this mimetic soil exactly reflects the real soil.

As shown in Fig. $3 \sum \Delta \mathrm{F}_{\mathrm{i}}$ is approximated area between two curves. If the ideal solution of optimization exists, the simulation curve coincides with measurement curve, i.e., no area exists.

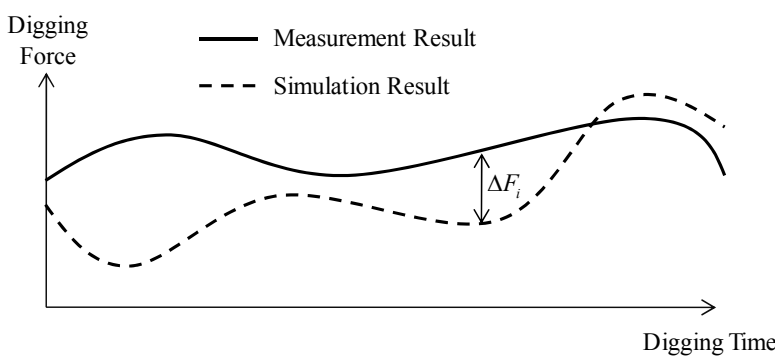

Step 1) Obtain $\Delta F_{i}(\mathrm{i}=1,2, \cdots, \mathrm{n})$ from divid ing total digging time by $\mathrm{n}$. Step 2) Find soil parameters such that minimize the value of $\sum \Delta F_{i}$

Fig. 3 Scheme for estimation of soil parameters

Even though there is no ideal solution, appropriate solution which is enough converged soil parameters can be useful and stored to soil database and reused in other simulations.

\section{INVERSE DYNAMICS OF LOAD WORK}

\subsection{INVERSE DYNAMICS}


Inverse dynamics is finding joint torque from position, velocity and acceleration of each joint. Since solving problems with closed chain is not easy, we cut the closed chains as seen in Fig. 4 and adapt open chain recursive dynamics algorithm.

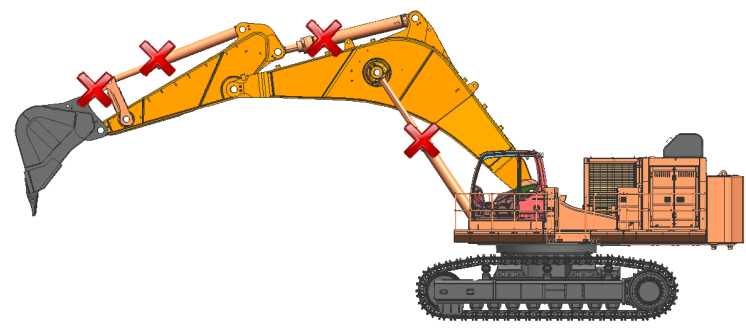

Fig.4 Reduced system of excavator

Dynamic formulation can be stated as below.

$$
\hat{\tau}=\tau+J^{T} F=M(q) \ddot{q}+C(q, \dot{q})
$$

$M$ is the mass matrix which is constituted as moment of inertia and mass. $C$ implies centripetal and coriolis forces. $J^{T} F$ is the external force of excavator which is the constraint force for compensating virtual open chain. $\tau$ is the joint moment, $q$ is the configuration matrix. In other words, inverse dynamics is calculating $\tau$ for given cylinder motion. The description of how to calculate constraint force is stated as below. [5]

If we let reduced system torque as $\hat{\tau}$, the relation of original system torque $\tau$ yields

$$
\hat{\tau}=\tau+J^{T} \lambda
$$

Constraint jacobian $J$ satisfies the relation of

$$
V=J \dot{q}
$$

$V$ is the velocity at the cutting face and the $q$ means the configuration of every joint. Since there are active and passive joints, taking just part of the passive ones the equation can be reordered as

$$
\begin{aligned}
& \hat{\tau}_{a}=\tau_{a}+J_{a}^{T} \lambda_{a} \\
& \hat{\tau}_{p}=\tau_{p}+J_{p}^{T} \lambda_{p}
\end{aligned}
$$

Since the passive joint torque of original system $\tau_{p}=0$,

$$
\lambda=J_{p}^{-T} \hat{\tau}_{p}
$$

Using $\lambda$ and $\tau$, torque of original system can be calculated.

\subsection{DIGGING WORK}

To calculate inverse dynamics of excavator in digging work, we apply digging force to it. The dynamic equation of the excavator is below form.

$$
M \ddot{q}+C=\tau+J^{T} F
$$

Our purpose in the sense of inverse dynamics is to obtain cylinder force $\tau$. For given excavator motion $q, \dot{q}, \ddot{q}$ we should know external force $F$ to get cylinder force $\tau$. In digging work $F$ is digging force exerted on bucket tooltip and can be calculated by using soil-tool interaction model. The digging force $F$ includes soil weight, soiltool friction force and soil moments.

\subsection{LIFTING WORK}

In lifting work inverse dynamics has some different procedure from the digging work. The soil volume on the bucket during digging work can be obtained by integration on the digging path as seen in Fig.5. Then soil density and volume determine the weight of soil on the bucket in lifting work.

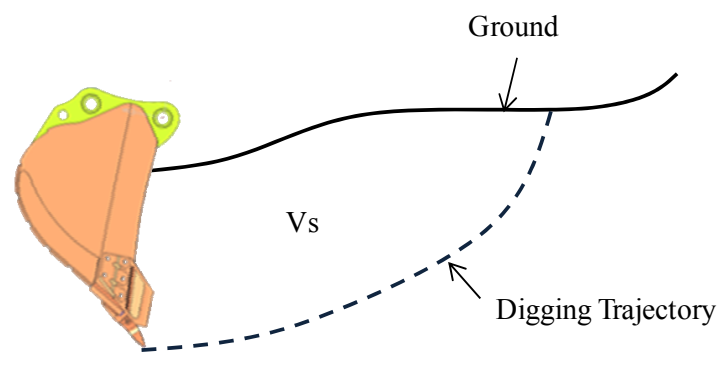

Fig.5 Digging trajectory and lifting volume (Vs)

In order to consider loading effect we add this weight to bucket. So the soil weight can be regarded as the additional bucket weight in lifting work. The dynamic equations are

$$
\hat{M} \ddot{q}+C=\tau
$$

$\hat{M}$ is the mass term considering additional bucket mass. There are no external forces in this work. Through this procedure we can get cylinder force $\tau$.

\subsection{DUMPING WORK}

In dumping work we assume that soil volume on the bucket is linearly decreases as the bucket angle increases as shown in Fig. 6. 


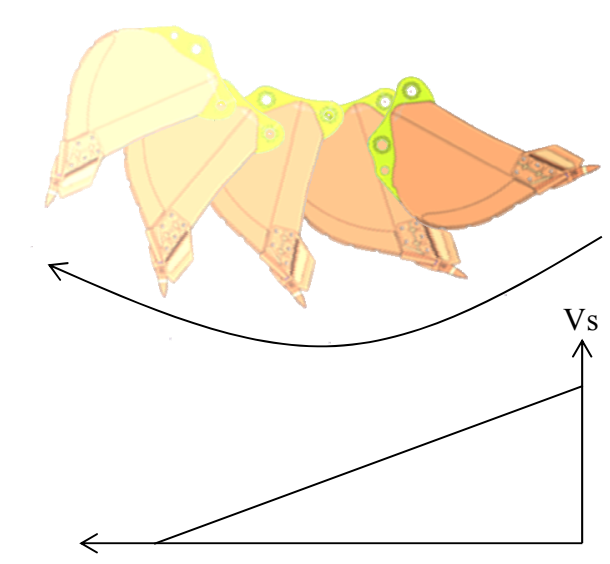

Bucket Angle

Fig.6 Soil volume on the bucket in dumping work

The dynamic equations are

$$
\hat{M}(\alpha) \ddot{q}+C=\tau
$$

$\hat{M}(\alpha)$ is the mass matrix and $\alpha$ is bucket angle. Because $\hat{M}(\alpha)$ is function of $\alpha$, implicitly $\hat{M}(\alpha)$ is also function of $q$, kinematic factor $\alpha$ should change the dynamic factor mass matrix. There are also no external forces in this work.

\subsection{VERIFICATION}

In Fig. 7 and 8 we compare measurement and simulation cylinder forces and swing torque data for loading existing or not. If there are no loading force, resultant forces is very similar. The correlation value is about 0.87 and the errors are due to mechanical friction and inaccurate mass data. If there are loading force, the accuracy of simulation decreases, however, relative magnitude of two cases is not largely different and enough meaningful.

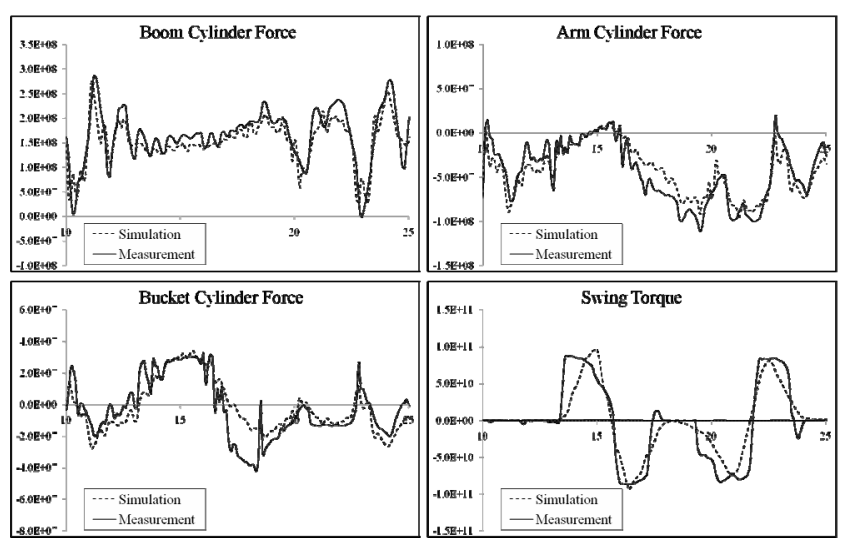

Fig.7 Cylinder force comparison with no loading

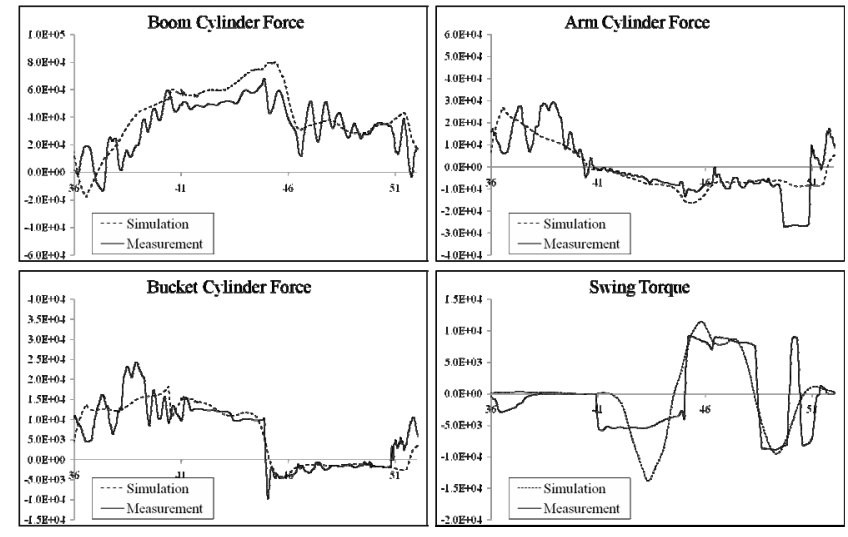

Fig.8 Cylinder force comparison with loading

\section{OPTIMAL PATH PLANNING}

An unmanned excavator should be able to generate a proper motion. To generate a motion, the excavator has to fill up the motion between initial and final positions. We can see this as a path planning problem since the purpose of path planning is to find a feasible path from given initial state to final state.

Generally in a mechanical system considering dynamics, state denotes both position and velocity. So a path planning problem should have initial and final positions and velocities as boundary conditions. There are infinitely many paths which satisfy these boundary conditions so you should choose the best one among these. We can define the best path as the optimal path and the optimal path means one that minimizes or maximizes some given objective function. Since optimal path varies with objective functions, there exist many optimal paths.

\subsection{OPTIMIZATION}

Generating optimal path can be considered as an optimization problem. To define optimization problem, you need to determine optimization variables and objective function and constraint functions. Here, optimization variable is not the path itself since path is infinite dimensional vector space. To solve the problem with numerical methods, the number of optimization variables should be finite. So we parameterize paths as B-splines and four B-splines denote three cylinder trajectories and one swing joint trajectory. Therefore control points and final time in B-splines become optimization variables.

Constraints vary with the excavator motion. When 
excavator is digging, a constraint should guarantee that digging volume is more than certain volume. When excavator is moving bucket to certain target point, a constraint which guarantees no penetration between bucket and ground is required. There exist more constraints following the characteristics of mechanical system such as flow rate limitation and cylinder pressure limitation. All constraints are as follows. Optimization problem can be written as follows.

$$
\begin{gathered}
\min _{x} L(x) \\
\text { subject to } g(x)=0 \text { and } h(x)<0
\end{gathered}
$$

Here, $x$ is set of control points in B-splines and $g(x)$ and $h(x)$ are constraints functions.

\subsection{EXCAVATOR MOTION PLANNING}

Optimal motion planning is the basic research on unmanned excavating system. The user just decides initial and final positions of the work trajectory, and the excavator should be able to generate motions. In this research, we choose minimum torque and minimum time as an objective function and that constrained by maximum pump flow rate, velocity constraints, final time, and maximum pressure of cylinder. The trajectories are interpolated by cubic Bsplines with 7 control points.

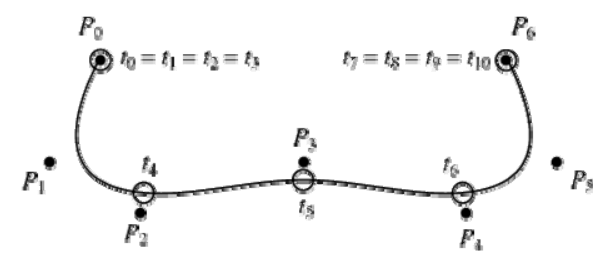

Fig.9 B-spline with 7 control points

\subsection{OBJECTIVE FUNCTION: MINIMUM TORQUE}

Minimum torque mode minimizes cylinder forces and swing torque. The problem is as follows.

$$
\min _{x} \int_{o}^{t f} \tau^{T} W \tau d t
$$

In Fig.10, 'flow', 'pressure' and 'no penetration between bucket and ground' constraints are applied. The calculated motion tends to keep the arm inner while moving because it saves swing torque.
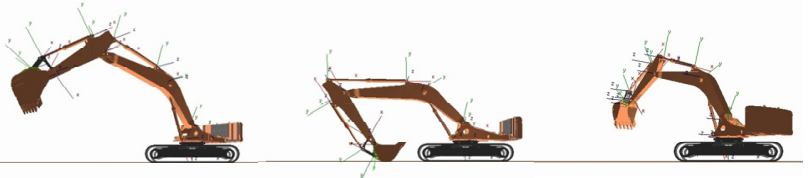

Fig.10 Calculated motion of minimum torque mode

\subsection{OBJECTIVE FUNCTION: MINIMUM TIME}

Minimum time mode minimizes total time spent to go to the final state. The problem is as follows.

$$
\min _{x, t f} \int_{o}^{t f} d t
$$

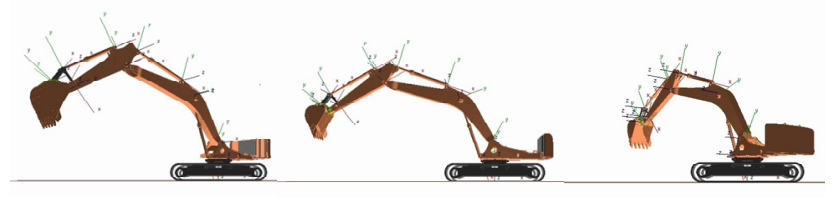

Fig.11 Calculated motion of minimum time mode

The calculated motion tends to make linear movement to go to the goal as fast as possible.

Minimum time mode minimizes the final time during excavating, which increase the efficiency of the excavating work. And minimum torque mode helps the user to save energy by reducing cylinder force that actuate the system, and guarantee better maintenance. Since the system shows high nonlinearity, minimum time reaches many local minima. We suggest another method for minimum time optimization. By optimizing torque, we reduce the given final time using line search. This method reduces the possibility of falling in local minima, and solves the problem more stable. The optimization problem is stated below.

1. First, solve $\min _{x} \int_{o}^{t f} \tau^{T} W \tau d t$ for given final time $t_{f}$.

2. If solution exists, reduce $t_{f}$ and try step.1 again.

3. If solution doesn't exist, the motion which is obtained from last step 1 is the optimal motion .

Generally minimum time solution tends to show bang-bang control which makes the system highly vibrate. But in suggested approach, we can avoid bang-bang control and get a stable motion. 


\subsection{COMPARISON WITH MEASUREMENTS}

To evaluate our optimization results, we need to compare the results with motions that skillful operator made. For this, we measured real motions of excavator which is made by skillful operator. The measurement data contains cylinder lengths, forces, swing angle, and swing torque. We set initial and final states as the same as measured motions and performed motion optimization.

The measured motion has a series of several tasks such as digging, lifting, dumping, and returning. So we cut this into 4 motion segments and solved 4 optimization problems with initial and final positions of each segments. We set initial and final velocities zero in optimization to make the problem simple.

As shown in Fig. 11 and 12, calculated digging motion seems quite similar to the measured motion but dumping and returning motions are different from the measured motion.

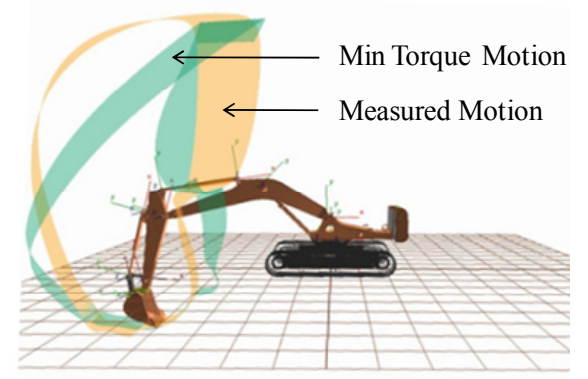

Fig.11 Comparison between min torque motion and measured motion

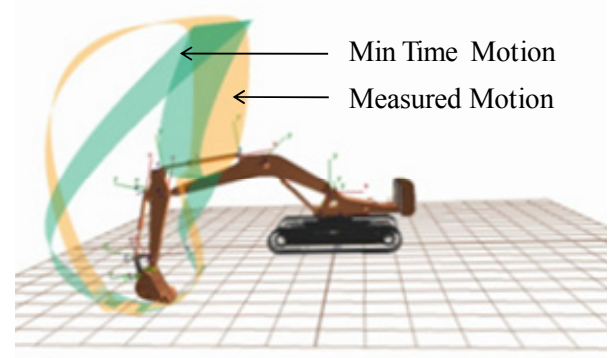

Fig.12 Comparison between min time motion and measured motion

The calculated dumping and returning motions seem to be more linear than measured motions because optimized motions are converged to save energy or time.

\section{CONCLUSIONS}

Our dynamics simulation shows high accuracy and this means solving inverse dynamics of mechanical system with several closed chains works well and our modeling of excavator is quite accurate. But digging forces show slight differences between real and simulation since our soil-tool interaction model is not perfect and some conditions of real digging motion are unknown.

On the other hand, we can generate excavator trajectory using only initial and final positions and simulate this motion in a virtual environment. It needs to be modified to account for more accurate hydraulic system but we expect that durability and performance of an excavator can be evaluated easily with this virtual digging simulation system.

\section{REFERENCES}

[1] Stentz, A., Bares, J., Singh, S. and Rowe, P., "A Robotic Excavator for Autonomous Truck Loading”, In Proceedings of the IEEE/RSJ International Conference on Intelligent Robotic Systems, 1998.

[2] Yamamoto, H., Moteki, M., Shao, H., Ootuki, T., Kanazawa, H. and Tanaka, Y., "Basic Technology toward Autonomous Hydraulic Excavator", $26^{\text {th }}$ International Symposium on Automation and Robotics in Construction, 2009.

[3] Reece, A.R., "The Fundamental Equation of Earthmoving Mechanics”, Proceedings of Institution of Mechanical Engineers, 1964.

[4] Singh, S., Cannon, H. and Luengo, O. "Modeling and Identification of Soil-tool Interaction in Automated Excavation”, In Proceedings, IEEE/RSJ International Conference on Intelligent Robotic Systems, 1998.

[5] Park, F.C., Choi, J. H. and Ploen, S. R., "Symbolic Formulation of Closed Chain Dynamics in Independent Coordinates", Mechanism and machine Theory, Vol. 34(5), pp. 731-751, 1999. 